\title{
Erratum to: Genetic and biochemical characterization of rhizobacterial strains and their potential use in combination with chelants for assisted phytoremediation
}

\author{
Angela Cicatelli $^{1} \cdot$ Francesco Guarino $^{1} \cdot$ Enrico Baldan $^{2} \cdot$ Stefano Castiglione $^{1}$
}

Published online: 24 February 2017

(C) Springer-Verlag Berlin Heidelberg 2017

Erratum to: Environ Sci Pollut Res

DOI 10.1007/s11356-016-7982-5

The original publication of this paper contains a mistake.

Author names were inadvertently interchanged.

The original article was corrected.

The online version of the original article can be found at http://dx.doi.org/ 10.1007/s11356-016-7982-5.

\section{Stefano Castiglione}

scastiglione@unisa.it

Angela Cicatelli

acicatelli@unisa.it

Francesco Guarino

fguarino@unisa.it

Enrico Baldan

enrico.baldan1@gmail.com

1 Dipartimento di Chimica e Biologia “A. Zambelli”, Università di

Salerno, Fisciano, 84084 Salerno, Italy

2 Dipartimento di Biologia, Università di Padova, 35131 Padova, Italy 